\title{
Strikingly Different Behaviors of Photoluminescence and Terahertz Generation in InGaN/GaN Quantum Wells
}

\author{
Guan Sun, Ruolin Chen, Yujie J. Ding, Fellow, IEEE, Hongping Zhao, Guangyu Liu, Jing Zhang, \\ and Nelson Tansu, Senior Member, IEEE
}

\begin{abstract}
We have investigated photoluminescence (PL) and terahertz $(\mathrm{THz})$ generation simultaneously from multiple InGaN/GaN quantum wells (QWs) with different well periods. The PL intensity fully saturates when the period of QWs is increased up to 4. However, THz output power continuously scales up even if the period of QWs is increased up to 16. Such a behavior indicates that high-power $\mathrm{THz}$ wave can be generated without efficient recombination of the photogenerated carriers, since $\mathrm{THz}$ is only generated during the absorption process. Following the measurements of intensity and peak energy of PL together with output power and spectra of $\mathrm{THz}$, we have concluded that the screening effect induced by photo-generated carriers can be neglected when the pump fluence is as low as $85 \mu \mathrm{J} / \mathrm{cm}^{2}$.
\end{abstract}

Index Terms-Broadband terahertz (THz) wave, InGaN/GaN quantum wells $(\mathrm{QWs})$, built-in field, dipole radiation, photoluminescence (PL).

\section{INTRODUCTION}

I NGAN/GAN quantum wells (QWs) have attracted a lot of attention due to their potential applications in light-emitting devices covering the regions from blue to green [1]-[3]. Moreover, it was proposed that a quantum cascade laser based on nitrides could be operated at room temperature [4]. Perhaps, the most unique property of InGaN/GaN QWs is the presence of a strong built-in electric field in the order of several megavolts per centimeter originating from piezoelectric polarizations if the

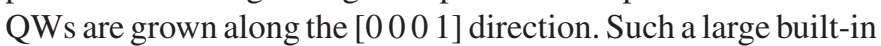
electric field induces a so-called quantum-confined Stark effect (QCSE), resulting in a significant separation of the electrons and holes generated in the InGaN/GaN QWs [5]-[7]. Due to such QCSE, the recombination rate is reduced since the poor overlap of wave functions of electron and hole. Obviously, for applications of laser diodes or light-emitting diodes, such electric field is an obstacle for achieving high efficiency or low

Manuscript received April 6, 2012; revised May 26, 2012; accepted July 2, 2012. Date of publication October 25, 2012; date of current version February 1, 2013. This work was supported by the Defense Advanced Research Projects Agency through the Air Force Research Laboratory and the National Science Foundation (ECCS 1028490).

G. Sun, R. Chen, Y. J. Ding, G. Liu, J. Zhang, and N. Tansu are with the Department of Electrical and Computer Engineering and Center for Optical Technologies, Lehigh University, Bethlehem, PA 18015 USA (e-mail: gus208@lehigh.edu; ruc210@lehigh.edu; gul308@lehigh.edu; jiz209@lehigh.edu; yud2@lehigh.edu; tansu@lehigh.edu).

H. Zhao is with the Department of Electrical Engineering and Computer Science, Case Western Reserve University, Cleveland, OH 44106 USA (e-mail: hongping.zhao@case.edu).

Color versions of one or more of the figures in this paper are available online at http://ieeexplore.ieee.org.

Digital Object Identifier 10.1109/JSTQE.2012.2218093 threshold devices. Therefore, in the past researchers have considered employing novel QWs structures in order to reduce the charge-separation effect [8], [9]. On the other hand, the built-in electric field in semiconductor bulk materials or nanostructures can be exploited for implementing certain devices without applying an external field. Indeed, terahertz $(\mathrm{THz})$ radiation was generated from a GaAs surface being illuminated by ultrafast laser pulses due to the screening of surface depletion field by virtual photo-generated carriers [10]. In the past, THz emission from GaAs/AlGaAs QWs with an external electrical field being present was observed with its mechanism being attributed to creation of polarized electron-hole pairs by ultrafast laser pulses [11]. Recently, microwatt $\mathrm{THz}$ pulse was generated in an eight-period $\operatorname{In}_{0.25} \mathrm{Ga}_{0.75} \mathrm{~N} / \mathrm{GaN}$ multiple QWs grown along $\left[\begin{array}{llll}0 & 0 & 0 & 1\end{array}\right]$ direction by utilizing its internal field [12]. It was pointed out that for high pump fluence, screening effect induced by photo-generated carriers plays a significant role in $\mathrm{THz}$ generation process. Moreover, it is believed to be the main mechanism for saturation of $\mathrm{THz}$ output power generated in InGaN/GaN QWs [13], [14].

In this paper, we report our results obtained on $\mathrm{InGaN} / \mathrm{GaN}$ QWs. Indeed, we simultaneously measured the photoluminescence (PL) and THz output in the InGaN/GaN QWs with different well periods. We have observed that the PL output was saturated when the period of QWs was increased up to four. However, the THz output scaled up more than linearly even with the period of the QWs increased up to 16. Through the comparison of experimental results of $\mathrm{PL}$ and $\mathrm{THz}$ measurements made on InGaN QWs with different well periods, we have concluded that high-power $\mathrm{THz}$ can be generated in InGaN QWs in spite of high density of nonradioactive structure defect. Such a conclusion is consistent with our proposed mechanism of $\mathrm{THz}$ generation in InGaN QWs, i.e., instantaneous generation of spatially separated electron-hole pairs resulting in efficient dipole radiation. Moreover, by combining the PL result with the $\mathrm{THz}$ signal, we have concluded that the screening effect induced by the photo-generated carriers is negligible for a pump fluence as low as $85 \mu \mathrm{J} / \mathrm{cm}^{2}$.

\section{DESCRIPTION OF SAMPLES AND EXPERIMENTAL SETUP}

InGaN/GaN QWs structures were grown on a 2.8- $\mu$ m-thick unintentionally doped $\mathrm{GaN}$ template (the background electron density of $\sim 4 \times 10^{16} \mathrm{~cm}^{-3}$ ) on a c-plane sapphire substrate by metal-organic chemical vapor deposition (MOCVD). The growth of the $\mathrm{GaN}$ template was performed at $1080{ }^{\circ} \mathrm{C}$ by 


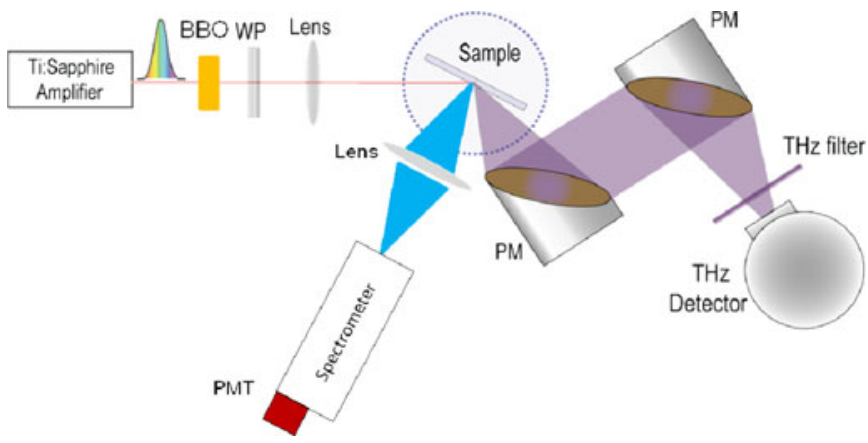

Fig. 1. Experimental setups used in the investigation of $\mathrm{THz}$ generation from InGaN/GaN quantum wells. WP: half-wave plate; PM: off-axis parabolic mirror; PMT: Photomultiplier. THz filters were used to block the residual pump laser beams while allowing the transmission of the $\mathrm{THz}$ waves.

employing 35-nm-thick low-temperature-grown $\left(T_{g}=525^{\circ} \mathrm{C}\right)$ GaN buffer layer. Subsequently, $\operatorname{In}_{0.19} \mathrm{Ga}_{0.81} \mathrm{~N} / \mathrm{GaN}$ QWs with different well periods ranging from 1 to 16 were deposited on the GaN template by MOCVD at $700{ }^{\circ} \mathrm{C}$. The thicknesses of the InGaN well and GaN barrier layers are 3 and $10 \mathrm{~nm}$, respectively. Broadband $\mathrm{THz}$ pulses were generated by using a coherent radiation beam at the wavelength of $391 \mathrm{~nm}$ after frequency-doubling the output beam from a Ti:sapphire regenerative amplifier, see Fig. 1 for the experiment setups. The pulse duration is measured as $210 \mathrm{fs}$ and repetition rate is $250 \mathrm{kHz}$. The excitation beam was then focused on the top surface of InGaN/GaN QWs with the laser spot area being measured to be about $0.85 \mathrm{~mm} \times 2.61 \mathrm{~mm}$. The polarization of laser was adjusted to be in the horizontal direction by using a half-wave plate, and incident angle was set as $70^{\circ}$ to minimize the reflection of the pump beam. The $\mathrm{THz}$ radiation was collimated, and then, focused onto a 4.2-K Si bolometer or pyroelectric detector by a pair of gold-coated parabolic mirrors in reflection geometries. Simultaneously, the PL of the InGaN QWs, excited by the same pump beam, was collected by a lens and then measured by a photomultiplier (PMT) after passing through a single-channel high-resolution spectrometer.

\section{RESULTS AND DISCUSSIONS}

In this section, we present our results obtained on the $\mathrm{THz}$ generation and PL from the $\operatorname{In}_{0.19} \mathrm{Ga}_{0.81} \mathrm{~N} / \mathrm{GaN} \mathrm{QWs}$ with different periods.

We have first investigated the PL spectra of each sample under room temperature with the pump fluence being set to $85 \mu \mathrm{J} / \mathrm{cm}^{2}$. As shown in Fig. 2(a), the emission spectrum measured on each sample peaks around $472 \mathrm{~nm}$. The multiple peaks modulation is caused by Fabry-Pérot resonance due to the multiple reflection caused by the front and back surfaces of each sample. From the spectra, it is clear that PL intensity scales up when the well period is increased up to four. However, when we further increase the well period, the PL intensity is significantly saturated, see Fig. 2(b). According to [15], we estimated the absorption length of the InGaN QW layers in our QW structure to be on the order of $100 \mathrm{~nm}$. Since the total thickness of the InGaN layers in the 16 periods of the InGaN/GaN QWs is $48 \mathrm{~nm}$, we have ruled out

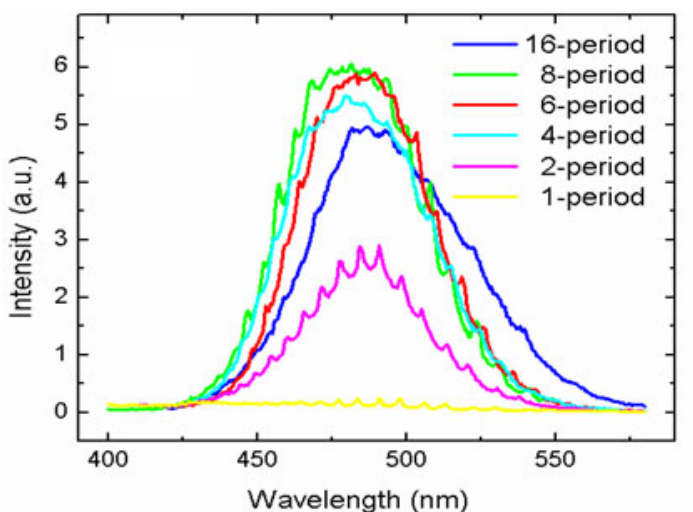

(a)

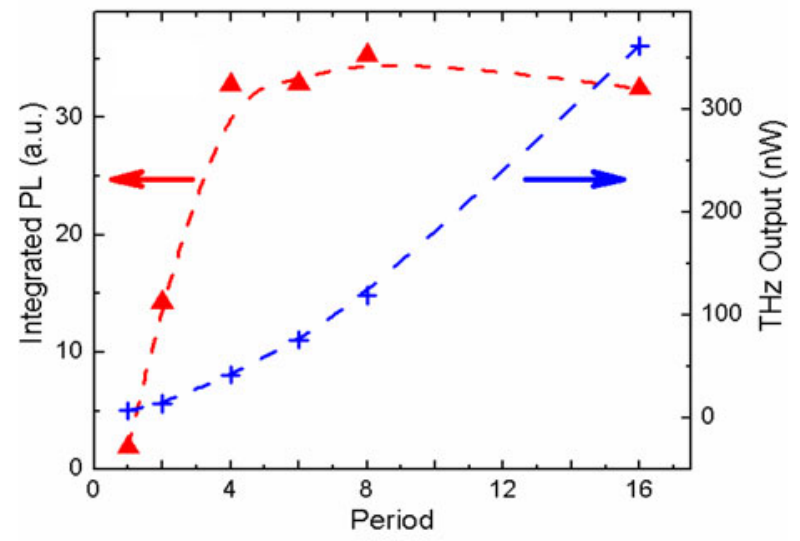

(b)

Fig. 2. (a) PL spectra of InGaN quantum wells with different periods measured at room temperature. (b) PL intensity and $\mathrm{THz}$ output power versus well period. Red triangular corresponds to PL intensity and blue cross corresponds to $\mathrm{THz}$ output power. The pump fluence is set as $85 \mu \mathrm{J} / \mathrm{cm}^{2}$.

the penetration saturation of the optical pumping as the mechanism for the PL saturation. In fact, for the 16-period sample, the transmitted pump beam is visible to the naked eyes. Such saturation could be caused by the increased density of the nonradiative structure defects. When the total thickness of the QWs scales up, the accumulated strain is expected to increase, which results in a higher density of nonradiative structure defects. It is worth noting that for the 16-period InGaN QWs, we have clearly observed submillimeter dots by naked eyes within the laser beam spot, especially on the edge of sample. These dots can be the indication that the total thickness of the InGaN/GaN QWs in this sample is close to the critical thickness.

For the THz generation, as illustrated by Fig. 2(b), the output power increases more than linearly even if the period of the QWs is increased up to 16 . The highest output power collected from the 16-period InGaN QWs is $360 \mathrm{nW}$. It is worth noting that the $\mathrm{THz}$ output power is not reduced when we move the laser spot onto the location where the sub-millimeter dots are observed. In our recent paper [12], we have attributed the mechanism for the $\mathrm{THz}$ generation to instantaneous creation of spatially separated electron-hole pairs resulting in efficient dipole radiation. It is well known that the output power for dipole radiation is proportional to square of dipole density. The density of the photogenerated carriers is proportional to the intensity of the 
optical pump. In addition, there are no phase differences among the THz waves generated by different QWs along the propagation direction. If we assume that the absorption is constant in each well, the THz output power should be quadratically dependent on the number of the QWs. The assumption that the phase difference of the generated $\mathrm{THz}$ wave in each $\mathrm{QW}$ is negligible makes sense since the total thickness of QWs is much smaller than the wavelength of the THz wave. Furthermore, unlike PL, the $\mathrm{THz}$ wave is generated during the absorption process not during the radiation process in the InGaN QWs. Since the nonradiative structure defects only influence the radiation process, the $\mathrm{THz}$ output power continuously increases with increasing the period of the QWs even if the PL exhibits saturation. Thus, in principle, we can further increase the periods of the InGaN QWs to further scale up the $\mathrm{THz}$ output power. The increase in the density of the nonradiative defects only influences the recombination process. For example, if we can add another 16 periods, the $\mathrm{THz}$ output power can be enhanced by a factor of 4. The increase in the indium composition in the wells can also be used to increase the $\mathrm{THz}$ output power since it causes electrons holes to further separate spatially, and therefore, the dipole strength can be further increased. Under the same experimental setup, an eight-period $\operatorname{In}_{0.25} \mathrm{Ga}_{0.75} \mathrm{~N} / \mathrm{GaN}$ QWs was measured, which shows less than a half of the PL intensity but nine times more $\mathrm{THz}$ output power than that of the eight-period $\mathrm{In}_{0.19} \mathrm{Ga}_{0.81} \mathrm{~N} / \mathrm{GaN}$ QWs described in this paper. Previously, it has been shown that by increasing the well width from 1.8 to $3.6 \mathrm{~nm}, \mathrm{THz}$ output power can be increased by $30 \%$ [13]. By optimizing the structure of the QWs, the THz output power is expected to increase to as high as $20 \mu \mathrm{W}$ under our experiment condition.

The dependence of the THz output power on the pump fluence for the 16-period InGaN QWs is shown in Fig. 3(a). Initially, for the pump fluencies up to $40 \mu \mathrm{J} / \mathrm{cm}^{2}$, the THz output power quadratically increases with the pump fluence, see the red fitting curve in Fig. 3(a). When the pump fluence is further increased up to $85 \mu \mathrm{J} / \mathrm{cm}^{2}$, a slight deviation to the quadratic fit is observed. Such a deviation has been attributed to the screening effect induced by the photo-generated electron-hole pairs and been supported by blueshift of the PL peak energy [13]. We have also plotted the peak energy of PL versus the pump fluence, see the blue dots of Fig 3(b). The peak energy for such 16-period InGaN QWs exhibits a blueshift in the amount of $60 \mathrm{meV}$, which appears to support the screening effect. However, according to the theoretical calculation [14], the screening effect increases the effective absorption coefficient, which is proportional to ratio of the integrated PL intensity with the pump fluence. Thus, based on such a calculation, we should expect that the integrated PL intensity scales up more than linearly when the pump fluence is increased. In experiment, apparently, the integrated PL intensity scales up less than linearly with the pump fluence, see the red triangles of Fig. 3(a), indicating that the absorption coefficient is actually reduced, which is not consistent with the screening effect. In a recent pump-probe experiment on similar InGaN QWs, the reduction of absorption has been observed for a pump fluence of as low as $0.19 \mathrm{~mJ} / \mathrm{cm}^{2}$ and explained by increased density of the hot carriers [16]. Thus, from the dependence of

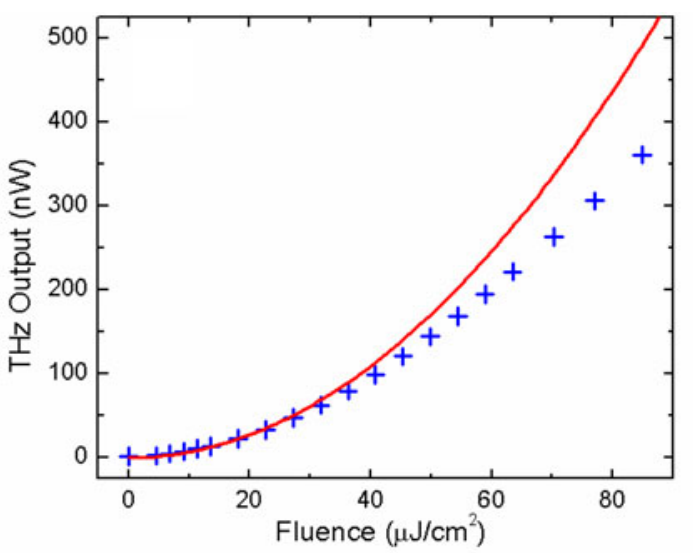

(a)

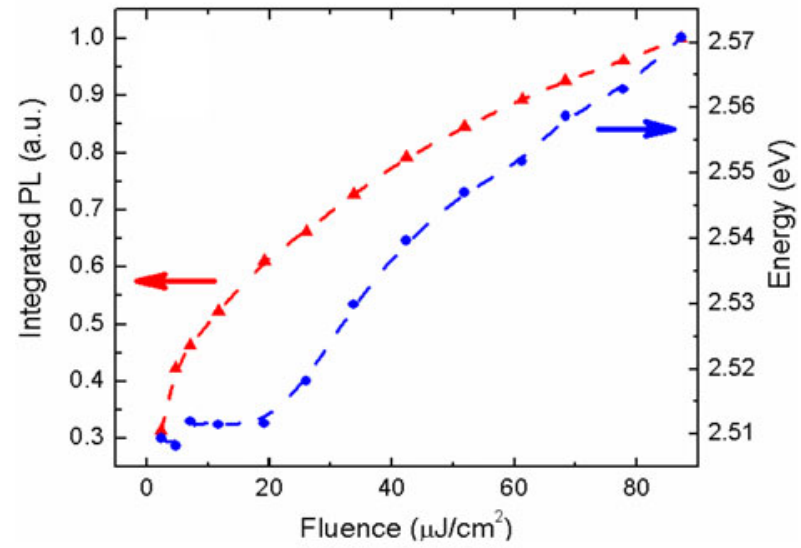

(b)

Fig. 3. (a) $\mathrm{THz}$ output power as a function of pump fluence. The crosses correspond to experiment data. The red curve corresponds to a quadratic fitting for first ten data points. (b) Integrated PL intensity and peak energy as a function of pump fluence. The red triangles and blue dots correspond to PL intensity and peak energy, respectively.

the integrated PL intensity on the pump fluence, we may draw a conclusion that the deviation or saturation is induced by the decrease in the absorption coefficient.

The contradicting conclusions from our analysis of the PL reveal that monitoring the PL signal may not be sufficient for us to understand the dynamic screening effect in the $\mathrm{THz}$ generation process, since $\mathrm{THz}$ is only generated during the absorption process whereas the PL is not only influenced by the absorption process but also by the recombination process. Indeed, the recombination mechanism is still not fully understood in the InGaN/GaN QWs. It is widely believed that the localized states play an important role in the emission process [17]-[19]. Such localized states induce a band-tail filling effect, resulting in the blue shift with increasing the pump power. Therefore, the blueshift for the InGaN QWs has been attributed to the combination of screening effect with band-tail filling effect [20]. In our detailed PL study on a similar InGaN sample, the dominant PL peak is explained by the recombination of the localized states [21]. Thus, we may over-emphasize the role of the screening effect if only monitoring the blueshift of PL peak.

An alternative method to determine the screening effect is to measure the spectra of the THz output from the InGaN QWs at 


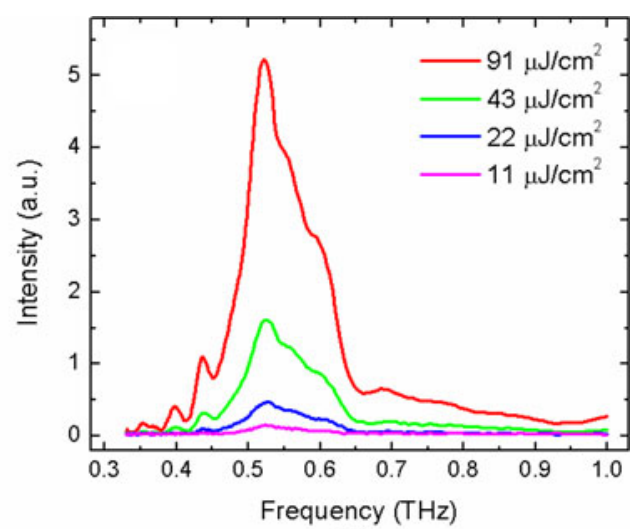

(a)

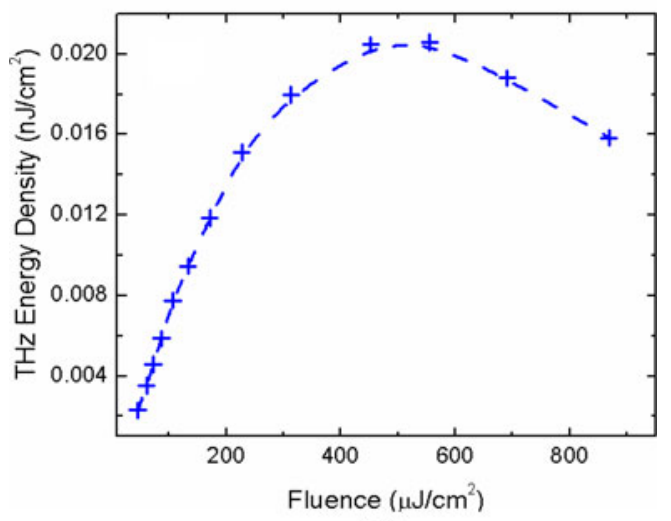

(b)

Fig. 4. (a) $\mathrm{THz}$ spectra measured at pump fluencies $11,22,43,91 \mu \mathrm{J} / \mathrm{cm}^{2}$. (b) THz energy density generated per well as function of pump fluence.

different pump fluencies. It has been pointed out in theory that dramatic broadening and shift of the $\mathrm{THz}$ spectra will be observed with increasing the pump fluence if dynamical screening is a strong absorption process [14]. Under such a predication, the $\mathrm{THz}$ spectral band width could far more exceed the bandwidth of the excitation laser. We have measured the THz spectra under several different pump fluencies by a homemade sub-millimeter diffraction grating system. Such a system allows us to detect whole $\mathrm{THz}$ spectra even if the bandwidth of the THz output is beyond the laser spectra, which is difficult to achieve in an electro-optic sampling system if the same laser is used to generate and sample the THz output. As is shown in Fig. 4(a), when the pump fluence is increased from 10 to $90 \mu \mathrm{J} / \mathrm{cm}^{2}$, within the accuracy of our measurements, we did not observe a bandwidth broadening or frequency shift of the $\mathrm{THz}$ spectra, which proves that in our experiment the screening effect induced by the photogenerated electron-hole pairs is negligible. It is worth noting that our conclusion is not in conflict with the work in [14], since the lowest fluence used in their calculation, which only causes a weak screening effect, is about two times as large as the highest value in our experiment.

In order to see a strong screening effect, we have reduced laser spot area to increase the pump fluence up to $1 \mathrm{~mJ} / \mathrm{cm}^{2}$. A full saturation behavior of the $\mathrm{THz}$ output power was observed when the pump fluence is about $0.6 \mathrm{~mJ} / \mathrm{cm}^{2}$. This saturation pump value is agreed with [16]. Because of the screening effect, the highest $\mathrm{THz}$ energy that can be generated in a single pulse is limited by the total static energy originally stored in the InGaN/GaN QWs. According to the simple capacitor model [13], the total energy stored in our InGaN QWs is estimated to be at least $100 \mathrm{~nJ} / \mathrm{cm}^{2}$. Experimentally, the saturation value for the $\mathrm{THz}$ energy density generated on each well is only about $0.02 \mathrm{~nJ} / \mathrm{cm}^{2}$, see Fig. 4(b), which is a factor of 5000 lower than the theoretical value. Thus, the screening effect is not the only mechanism to limit the $\mathrm{THz}$ output. A large amount $\mathrm{THz}$ output may not be collected in our experimental setup since a large fraction of the $\mathrm{THz}$ beam undergoes total internal reflection at the sample surface. Indeed, since the built-in field is always perpendicular to the sample surface, the most efficient radiation direction is along the surface, which can be hardly coupled out of the sample. In addition, we believe that free carriers inside the GaN template or photogenerated carriers inside the QWs also absorb a significant amount of the $\mathrm{THz}$ output power. Our simplified calculation indicates that the collected output power of $360 \mathrm{nW}$ obtained on the 16-period QWs corresponds to the total output power of about $60 \mu \mathrm{W}$ generated by the QWs. Previously, the increase in the absorption of the $\mathrm{THz}$ wave by the photogenerated carriers was investigated and evidenced in GaTe and InN materials [22], [23]. Such a mechanism can also explain the reduction of the $\mathrm{THz}$ output power when the pump fluence is higher than $0.6 \mathrm{~mJ} / \mathrm{cm}^{2}$, as shown in Fig. 4(b). Under the condition that the maximum degree of the screening effect is achieved, if increasing the pump fluence, more carriers are generated but there is no further increase in the THz output power. Since the more carriers only increase the absorption, the $\mathrm{THz}$ output power is reduced.

\section{CONCLUSION}

In conclusion, we have simultaneously investigated the PL and $\mathrm{THz}$ output from a set of the InGaN/GaN QWs with different periods. Since it is only generated during the absorption process, the $\mathrm{THz}$ output power continuously scales up with the number of the QWs. Thus, we can further increase the THz output power by adding up more periods of the QWs or increasing the indium composition in the well region. In contrast, the photoluminescence intensity may not increase. By measuring the $\mathrm{THz}$ spectra, we have concluded that for the pump fluence as low as $85 \mu \mathrm{J} / \mathrm{cm}^{2}$, the screening effect induced by the photo-generated carriers is negligible.

\section{REFERENCES}

[1] S. Nakamura, T. Mukai, and M. Senoh, "Candela-class high-brightness InGaN/AlGaN double-heterostructure blue-light-emitting diodes," Appl. Phys. Lett., vol. 64, no. 13, pp. 1687-1689, Jan. 1994.

[2] S. Nakamura, "The roles of structural imperfections in InGaN-Based blue light-emitting diodes and laser diodes," Science, vol. 281, no. 5379, pp. 956-961, 1998.

[3] S. Nakamura and G. Fasol, The Blue Laser Diode. Berlin, Germany: Springer, 1997.

[4] G. Sun, R. A. Soref, and J. B. Khurgin, "Active region design of a terahertz GaN/A10.15Ga0.85 N quantum cascade laser," Superlatt. Microstruct., vol. 37, no. 2, pp. 107-113, Feb. 2005.

[5] S. F. Chichibu, A. C. Abare, M. S. Minsky, S. Keller, S. B. Fleischer, J. E. Bowers, E. Hu, U. K. Mishra, L. A. Coldren, S. P. DenBaars, and 
T. Sota, "Effective band gap inhomogeneity and piezoelectric field in InGaN/GaN multiquantum well structures," Appl. Phys. Lett., vol. 73, no. 14, pp. 2006-2008, Jul. 1998.

[6] P. Perlin, C. Kisielowski, V. Iota, B. A. Weinstein, L. Mattos, N. A. Shapiro1, J. Kruger, E. R. Weber, and J. Yang, "InGaN/GaN quantum wells studied by high pressure, variable temperature, and excitation power spectroscopy," Appl. Phys. Lett., vol. 73, no. 19, pp. 2778-2780, Sep. 1998.

[7] T. Wang, J. Bai, S. Sakai, and J. K. Ho, "Investigation of the emission mechanism in InGaN/GaN-based light-emitting diodes," Appl. Phys. Lett., vol. 78, no. 18, pp. 2617-2619, Feb. 2001.

[8] R. A. Arif, H. P. Zhao, Y. K. Ee, and N. Tansu, "Spontaneous emission and characteristics of InGaN quantum wells light emitting diodes," IEEE J. Quantum Electron., vol. 44, no. 5/6, pp. 573-580, May/Jun. 2008.

[9] H. Zhao, R. A. Arif, and N. Tansu, "Design analysis of staggered InGaN quantum wells light-emitting diodes at 500-540 nm," IEEE J. Sel. Topics Quantum Electron., vol. 15, no. 4, pp. 1104-1114, Jul./Aug. 2009.

[10] B. B. Hu, X.-C. Zhang, and D. H. Auston, "Terahertz radiation induced by subband-gap femtosecond optical excitation of GaAs," Phys. Rev. Lett., vol. 67, no. 19, pp. 2709-2712, Nov. 1991.

[11] P. C. M. Planken, M. C. Nuss, W. H. Knox, D. A. B. Miller, and K. W. Goossen, "THz pulses from the creation of polarized electron-hole pairs in biased quantum wells," Appl. Phys. Lett., vol. 61, pp. 2009-2011, Oct. 1992

[12] G. Sun, G. Xu, Y. J. Ding, H. Zhao, G. Liu, J. Zhang, and N. Tansu, "Efficient terahertz generation within InGaN/GaN multiple quantum wells," IEEE J. Sel. Topics Quantum Electron., vol. 17, no. 1, pp. 48-53, Jan./Feb. 2011

[13] D. Turchinovich, P. Uhd Jepsen, B. S. Monozon, M. Koch, S. Lahmann, U. Rossow, and A. Hangleiter, "Ultrafast polarization dynamics in biased quantum wells under strong femtosecond optical excitation," Phys. Rev. B., vol. 68, no. 24, pp. 241307-1-241307-4, Dec. 2003.

[14] D. Turchinovich, B. S. Monozon, and P. Uhd Jepsen, "Role of dynamical screening in excitation kinetics of biased quantum wells: Nonlinear absorption and ultrabroadband terahertz emission," J. Appl. Phys., vol. 99, no. 1, pp. 013510-1-013510-8, Jan. 2006.

[15] R. Singh, D. Doppalapudi, T. D. Moustakas, and L. T. Romano, "Phase separation in InGaN thick films and formation of InGaN/GaN double heterostructures in the entire alloy composition," Appl. Phys. Lett., vol. 70, no. 9, pp. 1089-1091, Mar. 1997.

[16] P. J. S. van Capel, D. Turchinovich, H. P. Porte, S. Lahmann, U. Rossow, A. Hangleiter, and J. I. Dijkhuis, "Correlated terahertz acoustic and electromagnetic emission in dynamically screened InGaN/GaN quantum wells," Phys. Rev. B., vol. 84, no. 8, pp. 085371-1-085317-4, Aug. 2011.

[17] S. Chichibu, T. Azuhata, T. Sota, and S. Nakamura, "Spontaneous emission of localized excitons in InGaN single and multiquantum well structures," Appl. Phys. Lett., vol. 69, no. 27, pp. 4188-4190, Oct. 1996.

[18] Yong-Hoon Cho, G. H. Gainer, A. J. Fischer, J. J. Song, S. Keller, U. K. Mishra, and S. P. DenBaars, "S-shaped temperature-dependent emission shift and carrier dynamics in InGaN/GaN multiple quantum wells," Appl. Phys. Lett., vol. 73, no. 10, pp. 1370-1372, Sep. 1998.

[19] H. Schömig, S. Halm, A. Forchel, G. Bacher, J. Off, and F. Scholz, "Probing individual localization centers in an InGaN/GaN quantum well," Phys. Rev. Lett., vol. 92, no. 10, pp. 106802-1-106802-4, Mar. 2004.

[20] K. Kazlauskas, G. Tamulaitis, J. Mickevičius, E. Kuokštis, A. Žukauskas, Yung-Chen Cheng, Hsiang-Cheng Wang, Chi-Feng Huang, and C. C. Yang, "Excitation power dynamics of photoluminescence in In$\mathrm{GaN} / \mathrm{GaN}$ quantum wells with enhanced carrier localization," J. Appl. Phys., vol. 97, no. 1, pp. 013525-1-013525-7, Dec. 2004.

[21] G. Sun, G. Xu, Y. J. Ding, H. Zhao, G. Liu, J. Zhang, and N. Tansu, "Investigation of fast and slow decays in InGaN/GaN quantum wells," Appl. Phys. Lett., vol. 99, no. 8, pp. 081104-1-081104-3, Aug. 2011.

[22] G. Xu, G. Sun, Y. J. Ding, I. B. Zotova, K. C. Mandal, A. Mertiri, G. Pabst, R. Roy, and N. C. Fernelius, "Investigation of terahertz generation due to unidirectional diffusion of carriers in centrosymmetric GaTe crystals," IEEE J. Sel. Topics Quantum Electron., vol. 17, no. 1, pp. 30-37, Jan./Feb. 2011.

[23] G. Xu, Y. J. Ding, H. Zhao, G. Liu, M. Jamil, N. Tansu, I. B. Zotova, C. E. Stutz, D. E. Diggs, N. Fernelius, F. K. Hopkins, C. S. Gallinat, G. Koblmiller, and J. S. Speck, "THz generation from InN films due to destructive interference between optical rectification and photocurrent surge," Semicond. Sci. Technol., vol. 25, no. 1, pp. 015004-1-0150045, Jan. 2010.

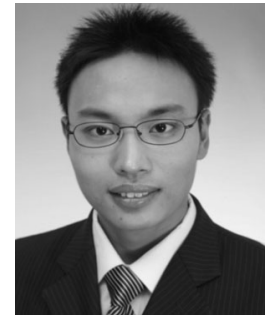

Guan Sun received the B.S. degree from Fudan University, Shanghai, China, in 2008. He is currently working toward the Ph.D. degree in the Department of Electrical and Computer Engineering, Lehigh University, Bethlehem, PA.

His research interests include terahertz generation and applications, photoluminescence, and $\mathrm{Ra}$ man scattering of III-nitride in terms of quantum wells and quantum dots.

Mr. Sun is a recipient of Dean's Scholarship (2008, lowship on Solid-State studies.

Lehigh University) and the Sherman-Fairchild Fel-

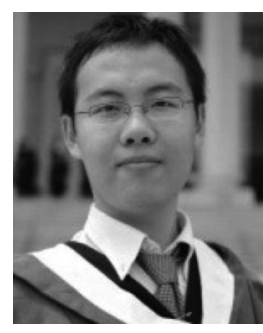

Ruolin Chen received the B.S. degree in physics from Fudan University, Shanghai, China, in 2010. He is currently working toward the Ph.D. degree in the Department of Electric and Computer Engineering, Lehigh University, Bethlehem, PA.

His current research interests include terahertz generation and applications, nonlinear optics, and IIInitride light emitting devices.

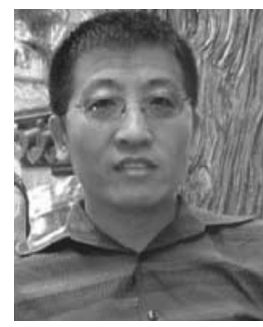

Yujie J. Ding (M'04-SM'05-F'13) received the B.S. degree from Jilin University, Changchun, China, in 1984, the M.S.E.E. from Purdue University, West Lafayette, IN, in 1987, and the Ph.D. degree from Johns Hopkins University, Baltimore, MD, in 1990.

During 1990-1992, he was a Postdoctoral Fellow, and later an Associate Research Scientist at Johns Hopkins University. During 1992-1999, he was an Assistant and Associate Professor of Physics at Bowling Green State University, Bowling Green, OH. During 1999-2002, he was an Associate Professor of Physics at the University of Arkansas, Fayetteville. In 2002, he joined Lehigh University, where he is currently a Professor in the Department of Electrical and Computer Engineering. He is the author or coauthor of about 160 refereed journal articles in optoelectronics, nonlinear optics, and quantum electronics. His current research interests include terahertz $(\mathrm{THz})$ generation, amplification and detection, nanostructures and nanodevices, resonance enhancements of Raman scattering in nitride heterostructures, and their applications.

Dr. Ding is a Fellow of the Optical Society of America. He received the Class of 1961 Professorship from Lehigh University, in 2003 and the Outstanding Young Scholar Award from Bowling Green State University in 1996.

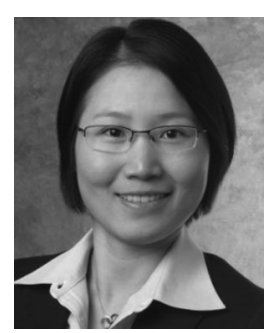

Hongping Zhao received the Bachelor's degree in physics from Nanjing Normal University, Jiangsu, China, in 2003, the Master's degree in electrical engineering from Southeast University, Jiangsu, in 2005 , and the $\mathrm{Ph} . \mathrm{D}$. degree in electrical engineering, Lehigh University, Bethlehem, PA, in 2011.

Since July 2011, she has been a tenure-tracked Assistant Professor in the Department of Electrical Engineering and Computer Science (EECS), Case Western Reserve University, Cleveland, $\mathrm{OH}$. She is the author or coauthor of more than 96 research publications in refereed international journals and conference proceedings. She is a regular reviewer of the leading journals in the areas of applied physics, quantum electronics, nanotechnology, photonics, and optoelectronics. Her research interests include device physics, epitaxial growth, and fabrication of semiconductor optoelectronics devices based on III-nitride semiconductor nanostructures.

Dr. Zhao received the SPIE Educational Scholarship in optical science and engineering for the years of 2008 and 2009. She also served as a panel review member for the National Science Foundation. 


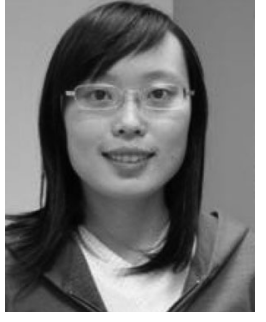

Guangyu Liu received the B.S. degree in electronic science and technology from the Huazhong University of Science and Technology, Hubei, China, in June 2008. Since July 2008, she has been working toward the Ph.D. degree in the Department of Electrical and Computer Engineering (ECE) and the Center for Optical Technologies (COT), Lehigh University, Bethlehem, PA.

Her research interests are related to III-Nitride semiconductor nanostructures and optoelectronics devices, covering the theoretical/computational, metalorganic chemical vapor deposition (MOCVD) growth, and device fabrication and characterization for high-performance III-Nitride light-emitting diodes (LEDs), III-Nitride quantum dots.

Ms. Liu is a recipient of SPIE Scholarship in Optics and Photonics (2012, Lehigh University), Sherman-Fairchild Fellowship (2012 and 2010, Lehigh University), the Dean's Scholarship (2008, Lehigh University) and Best Graduation Thesis (2008, Huazhong University of Science and Technology)

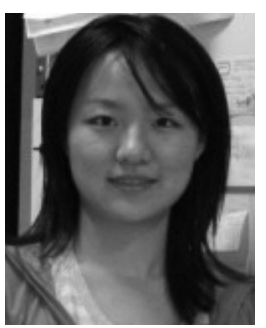

Jing Zhang received the B.S. degree in electronic science and technology from the Huazhong University of Science and Technology, Wuhan, China, in June 2009. Since August 2009, she has been working toward the Ph.D. degree in the Department of Electrical and Computer Engineering (ECE) and the Center for Optical Technologies (COT), Lehigh University, Bethlehem, PA

Her research areas are related to III-nitride semiconductor nanostructures for thermoelectric and solid state lighting technologies. Her research works cover various aspects of computational, growths, and device fabrication of III-nitride semiconductor for photonics and thermoelectric applications. Her research interests include the pursuit of novel materials for high thermoelectric figure of merit, deep Ultraviolet LEDs and lasers, as well as solid state lighting applications.

Ms. Zhang is a recipient of the Dean's Scholarship (2009, Lehigh University) and the Best Graduation Thesis (2009, Huazhong University of Science and Technology). She is two-times recipient of Sherman-Fairchild Fellowship for Solid State Studies (2011-2013, Lehigh University). She received the SPIE Scholarship in Optics and Photonics in 2012.

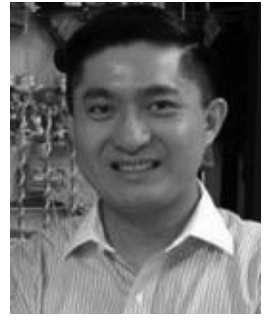

Nelson Tansu (SM'09) was born on October 1977, and received the B.S. degree (applied mathematics, electrical engineering, and physics; with highest distinction) and the Ph.D. degree (electrical engineering) from the University of Wisconsin-Madison, Madison, in May 1998 and May 2003, respectively.

He has been a faculty member at Lehigh University (Bethlehem, PA) since July 2003, and he is currently the Class of 1961 Associate Professor (with tenure) in the Department of Electrical and Computer Engineering (ECE) and Center for Optical Technologies (COT) at Lehigh University. His research works cover both the theoretical and experimental aspects of the physics of semiconductor optoelectronics materials and devices, the physics of low-dimensional semiconductor (nanostructure), and MOCVD and device fabrications of III-Nitride and III-V-Nitride semiconductor optoelectronics devices on $\mathrm{GaAs}$, InP, and $\mathrm{GaN}$ substrates. He has published in more than 220 refereed international journal (82) and conference publications $(140+)$, and he holds several U.S. patents (total $>8$ ).

Dr. Tansu was theWisconsin Alumni Research Foundation Graduate University Fellow and the Vilas Graduate University Fellow during his graduate studies at Wisconsin. He received the Graduate Dissertator Award at Wisconsin. Other selected awards include: the Harold A. Peterson Best ECE Dissertation Award (at Wisconsin), the 2008 Libsch Early Career Research Award (at Lehigh), and the 2010 Wisconsin Forward Under 40 for the Outstanding Young Alumn Award (at Wisconsin). He has also reviewed regularly with the leading journals in applied physics, quantum electronics, nanotechnology, photonics, and optoelectronics areas. He has also served several times as a panel member for the U.S. National Science Foundation (ECCS, DMR, STC Panel, ERC Panel/Site Reviewer, and SBIR), U.S. Department of Defense, U.S. Department of Energy, several other U.S. federal agencies, private foundations in U.S., and several funding agencies in Europe and Asia. Previously, he has also given numerous lectures, seminars, and invited talks (total $>40$ ) in universities, research institutions, and conferences in U.S., Canada, Europe, and Asia. He serves as the Primary Guest Editors of the IEEE JOURNAL OF SELECTED TOPICS IN QUANTUM ELECTRONICS (2008-2009) and the IEEE/OSA JOURNAL OF DISPLAY TECHNOLOGY (2012-2013), and he also serves as Associate Editors for IEEE PHOTONICS JOURNAL (2009-present), OSA Optical Materials Express (2011-present), and Nanoscale Research Letters (2007-present). He has also served as the Technical Program Committee for several major technical conferences for IEEE, OSA, SPIE, and APS; the selected lists include: IEEE/OSA Conference on Lasers and Electro-Optics (2007, 2008, 2009, and 2013), SPIE PhotonicsWest (20092013), APS March Annual Meeting (2007, 2009, and 2010), and others. He was also selected as Invited General Participants at the 2008 National Academy of Engineering (NAE)'s U.S. Frontiers of Engineering (USFOE) Symposium and the NAE's 2012 German-American Frontiers of Engineering Symposium (GAFOE), and he served as the Organizing Committee for the 2009 NAE's U.S. Frontiers of Engineering Symposium. 